\title{
THE PHASE OF SECOND-HARMONIC LIGHT GENERATED AT AN INTERFACE AND ITS RELATION TO ABSOLUTE MOLECULAR ORIENTATION
}

\author{
K. KEMNITZ, K. BHATTACHARYYA, J.M. HICKS, G.R. PINTO, K.B. EISENTHAL \\ Department of Chemistry, Columbia University, New York, NY 10027, USA
}

and

T.F. HEINZ

IBM T.J. Watson Research Center, Yorktown Heights, NY 10598, USA

Received 15 September 1986; in final form 14 October 1986

\begin{abstract}
Measurement of the absolute phase of the surface second-harmonic light field with respect to the pump field, i.e. the phase of the surface non-linear susceptibility, is described. From such studies the absolute orientation of surface molecules can be determined. In the present work the liquid/vapor interface of an aqueous phenol solution was investigated.
\end{abstract}

\section{Introduction}

The non-linear optical process of second-harmonic generation (SHG) possesses an intrinsic sensitivity to the asymmetry present at the interface between two centrosymmetric media. This property, arising from the fact that SHG is electric-dipole forbidden in the bulk [1], has permitted the technique to be applied in a variety of studies of surfaces and interfaces [2]. Among the systems that have been explored in this way are molecules adsorbed on solid surfaces [3-11], insoluble surfactant layers [12], liquid-solid interfaces [13-17] and, in our recent work, surface of liquids [18]. Valuable information on interfacial properties has been obtained from the measurements of the second-harmonic ( $\mathrm{SH}$ ) efficiency, polarization dependence, and frequency dependence. In the case of molecules at an interface, for example, these data can be related to molecular concentration [5,13], orientation [5] and spectroscopy [4]. However, to date, no experiment has been performed on the absolute phase of the elements of the surface non-linear susceptibility tensor ${ }^{*} \chi_{s}^{(2)}$, i.e. on the absolute phase of the SH electric fields with respect to the pump radiation.

In this paper, we describe a procedure for such absolute phase measurements and develop a scheme for inferring the absolute orientation of molecules at an interface with respect to the directed surface normal. This new information about the sense of molecular orientation, which cannot be obtained directly from conventional linear optical spectroscopy, should help to provide insight into the basic chemical interactions at surfaces and interfaces. The principle of the method can be understood simply. When the SH radiation arises from the electric-dipole response of an oriented layer of molecules, we can write the surface non-linear susceptibility (neglecting local-field effects [19]) as a sum of the non-linear polarizabilities $\boldsymbol{a}^{(2)}$ of the molecules:

$\chi_{\mathrm{s}}^{(2)}=N_{\mathrm{s}}\left\langle\boldsymbol{\alpha}^{(2)}\right\rangle$.

Here the sum over molecular polarizabilities has been expressed as an average of the polarizability over molecular orientations times the number of molecules per unit area, $N_{\mathrm{s}}$. From the tensor properties of the non-linear polarizability, we then see that performing an inversion operation on the molecules will

: The surface non-linear susceptibility tensor $\chi_{s}^{(2)}$ relates the nonlinear source polarization per unit area in the surface to the square of the electric field at the fundamental frequency. 
lead to a change in sign of the non-linear susceptibility. More specifically, for an arrangement of molecules isotropic in the surface plane, as one would find for a liquid, a reflection through the surface plane will cause the sign of the elements of $\chi_{\mathrm{s}}^{(2)}$ to switch. Thus, a comparison of the signs (or, more generally, phases) of the elements of $\boldsymbol{\alpha}^{(2)}$ with the signs of $\chi_{\mathrm{s}}^{(2)}$ will provide information on the absolute sense of the molecular orientation with respect to the directed surface normal.

We illustrate the utility of the phase measurements of $\chi_{s}^{(2)}$ in the case of a phenol molecular layer on the surface of an aqueous solution. In earlier work [18] we reported on the average orientation of the principal molecular axis (passing through the phenyl- oxygen bond) with respect to the surface normal. From the phase measurement, we can actually determine the sense of this principal axis with respect to the surface, namely, whether the phenyl-oxygen bond is directed towards or away from the bulk water. As one would anticipate for a hydrophilic moiety such as the hydroxy group, we find from our measurements that the phenyl-oxygen bond is directed towards the liquid solution. In the following section, we discuss the salient features of the phase measurement, both in general and for the case of the aqueous phenol solution. Subsequently, we present an analysis of the phases of the elements of the non-linear susceptibility in terms of the absolute molecular orientation and consider the implications for the phenol/water system.

\section{Measurement of the phase of the non-linear susceptibility}

\subsection{Method of phase measurement}

The scheme of the phase measurement, performed in the reflection geometry, is illustrated in fig. 1. As in the early studies on the phase of bulk non-linear susceptibilities by Bloembergen and co-workers [20], $\mathrm{SH}$ radiation is generated in a reference crystal by the fundamental beam reflected from the surface. After appropriate spectral filtering, we detect the intensity corresponding to the sum of SH fields from the sample and the non-linear reference. Information on the phase of the SH field from the sample is

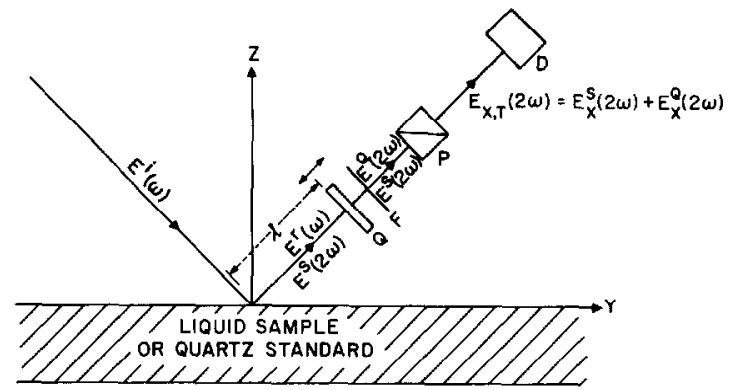

Fig. 1. Experimental scheme for phase measurements. Q, reference quartz; $P$, analyzer; D, monochromator and detector assembly. $E^{\prime}$ and $E^{r}$ are respectively, the incident and reflected fundamental field amplitudes; $E^{\mathrm{S}}$ and $E^{\mathrm{Q}}$, the $\mathrm{SH}$ fields generated by the sample (or quartz standard) and the reference quartz, respectively; $E_{\mathrm{r}}^{\mathrm{S}}, E_{x}^{\mathrm{Q}}$ and $E_{\mathrm{r}, \mathrm{T}}$, the $x$ components of the SH field due to the sample, reference, and their sum, respectively.

obtained from the interference between the two beams, which can be varied by translating the nonlinear reference along the path of the beam.

As the reference crystal is moved away from the sample by $\delta l$, the fundamental travels a greater distance and the path length for the $\mathrm{SH}$ radiation from the reference is decreased correspondingly. Taking into account the dispersion of the ambient air, we see that the phase of the reference SH wave is altered by

$$
\begin{aligned}
\delta \phi & =(2 \omega / c)[n(\omega)-n(2 \omega)] \delta l \\
& =-(2 \omega / c) \Delta n \delta l,
\end{aligned}
$$

where $\omega$ represents the fundamental frequency and $\Delta n=n(2 \omega)-n(\omega)>0$ denotes the difference in refractive indices of air at $2 \omega$ and $\omega$. Thus, by scanning the position of the quartz reference, we observe a modulation in the net $\mathrm{SH}$ intensity. A given phase of the surface non-linear susceptibility produces a particular interference pattern. For a surface nonlinear susceptibility with a more advanced phase, the positions of the maxima and the minima will appear shifted towards the sample, as specified by eq. (2).

In order to obtain the absolute phase of the surface non-linear susceptibility of the sample, comparison is made with a calibrated standard. For this purpose, only bulk non-linear materials are available; however, as can be readily seen by a superposition argument or directly from Maxwell's equations [21], the bulk material gives rise to a reflected SH wave with a phase advanced by $90^{\circ}$ with respect to that from 
an optically thin surface layer of the material ${ }^{*}$. For example, if the phase of the non-linear susceptibility of the bulk material is $180^{\circ}$, as for $\chi_{x x x}$ of righthanded quartz crystal discussed below, the observed pattern will correspond to a surface non-linear susceptibility with a phasc of $270^{\circ}$.

\subsection{Experimental procedure}

The experimental apparatus employed for these phase measurements on the surface of an aqueous phenol solution was essentially that described previously [18]. The excitation was provided by an amplified picosecond dye laser operating at $616 \mathrm{~nm}$. The fundamental beam was focused on the liquid surface at a $70^{\circ}$ angle of incidence. As before, the SH radiation from the surface was detected by photon counting techniques with a cooled photomultiplier. The SH light was isolated by means of appropriate color filters and a monochromator. In these phase measurements, the concentration of the phenol solution was $0.4 \mathrm{M}$, which yielded roughly a monolayer of phenol at the surface [18]. At this concentration the SH intensity from the phenol-covered surface was roughly ten times that of neat water, which indicates that the non-linear response was dominated by the contribution of phenol.

In the experiment with phenol, we choose to determine the phase of the $x z x$ component of the surface non-linear susceptibility, $\left(\chi_{s}^{(2)}\right)_{x z x}$, where $z$ corresponds to the outward surface normal and $x$ to a direction in the (isotropic) surface plane perpendicular to the plane of incidence of the laser light. The SH radiation associated with this tensor element could be isolated by means of the following arrangement of input and output polarizations. The pump electric field was adjusted to have both $\mathrm{s}$ and $\mathrm{p}$ components, with the polarization vector lying at $45^{\circ}$ from the plane of incidence and having positive projections along the $x$ and $z$ axes. The analyzer selected the s-polarized SH field for detection. Any higherorder (electric-dipole forbidden) non-linear polarization appearing in the bulk liquid will not contribute to the $\mathrm{SH}$ field measured in this configuration,

\footnotetext{
The equivalence presumes that the effects of birefringence and optical activity are insignificant and that the material is transparent.
}

since for an isotropic sample the bulk terms produce only p-polarized radiation [22].

The non-linear reference for the interference measurement was a thin z-cut quartz crystal. Quartz proved to be a convenient choice for the non-linear material for several reasons, including its transparency at both the fundamental and harmonic frequencies and its lack of birefringence. Another important consideration was the poor SHG efficiency of quartz associated with its short coherence length. This property, together with the low reflectivity of the fundamental beam from the liquid surface and its spatial divergence while propagating from the sample to the non-linear reference, led to very weak SHG from the reference. In this way, a SH wave from the bulk non-linearity of the reference was produced with an amplitude comparable to that from the surface layer of the liquid sample, as required to observe wellmodulated interference fringes. To producc the interference pattern between the $\mathrm{SH}$ waves from the sample and the non-linear reference, we moved the quartz reference along the path of the reflected beam. The expected period of this pattern is given by eq. (2) as

$l_{0}=\pi c / \Delta n \omega=\lambda / 2 \Delta n$,

where $\lambda=2 \pi c / \omega$ is the wavelength of the pump radiation. For our measurement with the non-linear reference surrounded by air, we find $l_{0}=22 \mathrm{~mm}$, a distance which could be easily scanned by means of a standard translation stage.

The absolute phase calibration was accomplished by replacing the liquid sample with a second, thick quartz sample. The positive $x$ axis of the right-handed $z$-cut crystal was identified by means of its piezoelectric properties $\#$, and aligned along the positive $x$ axis of the laboratory coordinate system. In order to access $\chi_{x x x x}^{(2)}$, the pump beam was s polarized. It should be noted that the polarization of the reflected pump beam was essentially unchanged from that of the reflection of the mixed polarized pump beam from the liquid surface. This property arises from the fact that reflection from the liquid surface occurred in the

* Following the IEEE convention [23] the positive $x$ axis for right-handed (left-handed) quartz is the direction of the outward surface normal of the face developing positive charge on compression (extension). The sign of $\chi^{(2)}$ is then positive for left-handed quartz [24] and negative for right-handed quartz. 


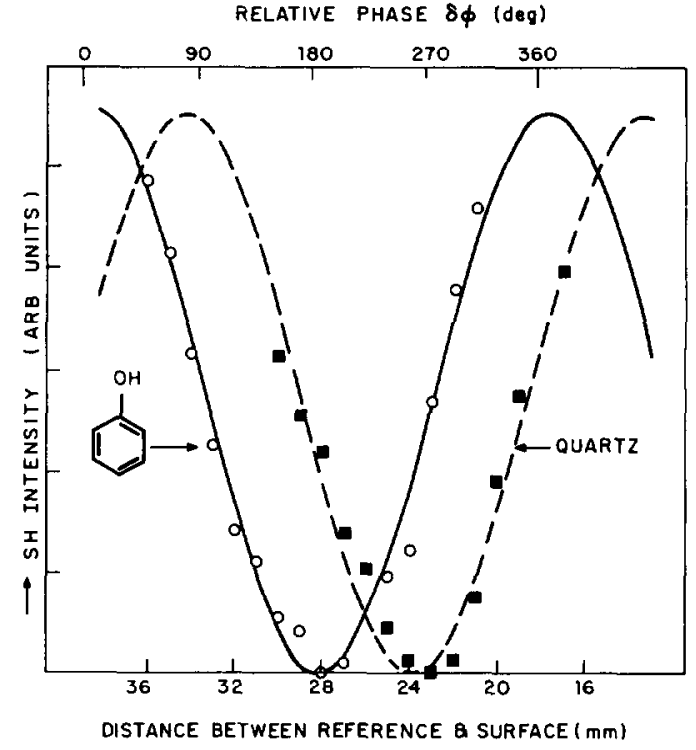

Fig. 2. Interference pattern of the SH fields for the surface of a $0.4 \mathrm{M}$ aqueous phenol solution (open circles) and for the quartz standard (filled boxes). The points are experimental data and the curves through them represent the best fits to $\cos ^{2}$ functions. The two interference patterns were rescaled to appear with comparable amplitudes.

vicinity of Brewster's angle, producing nearly spolarized light.

\subsection{Experimental results}

The interference patterns obtained by translating the non-linear reference are shown in fig. 2 . for both the surface of the aqueous phenol solution and for the quartz standard. The curves through the data points correspond to the expected $\cos ^{2}$ form for the SH intensity of two interfering electric fields. From repeated measurements, we found that the trace for the liquid surface is delayed by $90 \pm 15^{\circ}$ with respect to the quartz standard. As argued above, the absolute phase of $\left(\chi_{\mathrm{s}}^{(2)}\right)_{x=x}$ for the liquid is then $270^{\circ}-90^{\circ}=180^{\circ}$.

\section{Interpretation of phase measurement}

\subsection{General analysis}

We now consider the relation between the phases of the elements of $\chi_{s}^{(2)}$ and molecular orientation. For the sake of clarity and in keeping with the application to phenol, we shall analyze only the situation where $\chi_{s}^{(2)}$ is given as a sum of molecular non-linear polarizabilities with $\boldsymbol{\alpha}^{(2)}$ dominated by a single axial component $\alpha_{\xi \zeta \xi}^{(2)}$. We further suppose that the molecules are arranged isotropically with respect to the azimuthal directions. In this instance, the molecular orientation is characterized by the average value of the polar angle $\theta$ (or more precisely, by the distribution of polar angles) between the molecular $\zeta$ axis and the positive surface normal. Information on molecular orientation follows from an examination of the independent tensor elements of $\chi_{s}^{(2)}$ expressed in terms of molecular quantities. For the case at hand, we obtain the following explicit expressions from eq. (1):

$$
\begin{aligned}
& \left(\chi_{\mathrm{s}}^{(2)}\right)_{z=z}=N_{\mathrm{s}}\left\langle\cos ^{3} \theta\right\rangle \alpha_{\xi \zeta \xi}^{(2)}, \\
& \left(\chi_{\mathrm{s}}^{(2)}\right)_{x=x}=\left(\chi_{\mathrm{s}}^{(2)}\right)_{z x x} \\
& =\frac{1}{2} N_{\mathrm{s}}\left\langle\cos \theta \sin ^{2} \theta\right\rangle \alpha_{\zeta \zeta \xi}^{(2)},
\end{aligned}
$$

where $N_{\mathrm{s}}$ is the surface density of molecules and angular brackets signify an average over molecular orientations, as above.

In previous work on molecular orientation, relying on the polarization dependence of the SHG process $[5,12,18]$, only the ratio of these independent tensor elements could be found. With an absolute intensity calibration, the modulus of each element can also be determined. Only by means of a phase measurement, however, can the absolute signs of these quantities be established. Note that a reflection of all molecular orientations through the surface plane $(\theta \rightarrow \pi-\theta)$ changes the signs of $\left(\chi_{\mathrm{s}}^{(2)}\right)_{z z z}$ and $\left(\chi_{\mathrm{s}}^{(2)}\right)_{x z x}$ individually, but does not alter the sign of their ratio. In fact, for any isotropic surface, the components of $\chi_{s}^{(2)}$ will switch sign if the molecular orientations are reflected about the surface plane, but their ratios will remain unaffected. Thus the phase data can gencrally be related to the sense of the molecular axes with respect to the directed surface normal, provided that the surface non-linearity arises from an electric dipole response.

If the sign of $\alpha_{\xi \xi \xi}^{(2)}$ is known, either through calcu- 
lation or experiment [25], one can determine the direction of the positive molecular $\hat{\zeta}$ axis with respect to the surface normal $\hat{z}$. When $\left(\chi_{\mathrm{s}}^{(2)}\right)_{x x x} / \alpha_{\xi \zeta \xi}^{(2)}>0$, for example, $\hat{\zeta}$ is directed predominantly parallel to $\hat{z}$; conversely $\left(\chi_{\mathrm{s}}^{(2)}\right)_{x z x} / \alpha_{\zeta \zeta \xi}^{(2)}<0$ indicates that $\bar{\zeta}$ is oppositely directed to $\hat{z}$. The same relations apply, of course, to $\left(\chi_{\mathrm{s}}^{(2)}\right)_{z z i}$. When molecules point in both directions, the relative sign is determined by the appropriate weighted average.

\subsection{Phenol/water system}

Before we can apply the analysis developed above to the surface layer of the aqueous phenol solution, we must first establish that the non-linear response is electric-dipole in nature and, secondly, that the non-linear polarizability of phenol is dominated by the axial component $\alpha_{\xi \xi \xi}^{\{\xi}$. As has been recognized since the early observations of surface SHG [22], the presence of material boundaries can enhance the normally weak electric-quadrupole terms at the interface. To study the importance of these effects, we investigated the more symmetric benzene derivative $p$-dihydroxybenzene on the surface of an aqueous solution. Despite the greater importance of resonant response for these molecules as compared with the asymmetric phenol molecules, we observed a marked drop in SHG for the case of p-dihydroxybenzene. This strongly suggests that the intrinsic electric-dipole non-linearity of phenol accounts for most of the surface non-linearity. It should also be observed that our previous comparison [18] of surface density of phenol as a function of solute concentration from SHG data and from thermodynamic data indicates that the local-field effects (between surface solute molecules) are not significant .

In regard to the form of the non-linear polarizability of phenol, we refer to existing theoretical calculations [26]. In these studies, it was found that for phenol (as well as many other mono- and p-disubstituted benzene derivatives) $a^{(2)}$ is dominated by $\alpha\{\xi$, with $\zeta$ lying along the principal molecular axis. It was further established that $\alpha\{\xi \xi>0$ for $\hat{\zeta}$ directed from the center of the molecule to the oxygen atom. With this definition of the molecular $\zeta$ axis, the experimental data imply $\left(\chi_{\mathrm{s}}^{(2)}\right)_{x z x} / \alpha_{\xi \xi \xi}^{(2)}<0$. Hence, the molecular $\zeta$ axis is oriented oppositely to the outward surface normal, and we deduce that the sense of the phenol molecules is such that the hydroxy group points into the bulk liquid.

\section{Conclusion}

We have performed the first measurement of the absolute phase of a surface non-linear susceptibility. For a non-linear optical response describable as the sum of electric-dipole contributions from an oriented molecular layer, such phase data can be related to the absolute molecular orientation with respect to the directed surface normal. This information cannot be inferred directly from commonly used linear optical methods. In this work, we have demonstrated the non-linear technique for the case of molecules of phenol on the surface of an aqueous solution. Our results confirm the intuitive notion that the hydroxy group points towards the bulk water. A further point of interest is that when surface structural information is available, one can use the phase measurements, as described here, to obtain the phases of the elements of the molecular non-linear polarizability.

\section{Acknowledgement}

The authors would like to thank Dr. R.C. Miller for helpful comments concerning the sign of the nonlinear susceptibility of quartz and Dr. J.J. Wynne for a careful reading of the manuscript. The generous support of the National Science Foundation, the Air Force Office of Scientific Research, and the Joint Services Electronics Program 29-82-K-0080 is gratefully acknowledged.

\section{References}

[1] Y.R. Shen, The principles of nonlinear optics (Wiley, New York, 1984) ch. 25.

[2] Y.R. Shen, J. Vacuum Sci. Technol. B3 (1985) 1464

[3] J.M. Chen, J.R. Bower, C.S. Wang and C.H. Lee, Opt. Commun. 9 (1973) 132;

J.M. Chen, J.R. Bower and C.S. Wang, Japan. J. Appl. Phys. Suppl. 2 (1974) 711.

[4] T.F. Heinz, C.K. Chen. D. Ricard and Y.R. Shen, Phys. Rev. Letters 48 (1982) 478 . 
[5] T.F. Heinz, H.W.K. Tom and Y.R. Shen, Phys. Rev. A28 (1983) 1883.

[6] H.W.K. Tom, C.M. Mate, X.D. Zhu, J.E. Crowell, T.F. Heinz, G.A. Somorjai and Y.R. Shen, Phys. Rev. Letters 52 (1984) 348.

[7] X.D. Zhu, Y.R. Shen and R. Carr, Surface Sci. 163 (1985) 114.

[8] G. Marowsky, A. Giersulski and B. Dick, Opt. Commun. 52 (1985) 339, and references therein.

[9] P. Di Lazzaro, P. Mahatoni and F. de Martini, Chem. Phys. Letters 114 (1985) 103.

[10] Z. Chen, W. Chen, J. Zheng, W. Wang and Z. Zhang, Opt. Commun. 54 (1985) 305.

[11] N.E. van Wyck, E.W. Koenig, J.D. Bynes and W.M. Hetherington III, Chem. Phys. Letters 122 (1985) 153.

[12] Th. Rasing, Y.R. Shen, M.W. Kim, P. Valint and J. Bock, Phys. Rev. A31 (1985) 537;

Th. Rasing, Y.R. Shen, M.W. Kim, S. Grubb and J. Bock, Phys. Rev. Letters 55 (1985) 2903.

[13] C.K. Chen, T.F. Heinz, D. Ricard and Y.R. Shen, Phys. Rev. B27 (1983) 1965, and references therein.

[14] R.M. Corn, M. Romagnoli and M.D. Levenson, Chem. Phys. Letters 106 (1984) 30; J. Chem. Phys. 81 (1984) 4127.
[15] T.T. Chen. K.U. von Raben, D.V. Murphy, R.K. Chang and B.L. Laube, Surfacc Sci. 143 (1984) 369.

[16] G.L. Richmond, Langmuir 2 (1986) 132, and references therein.

[17] D.F. Voss, M. Nagumo, L.S. Goldberg and K.A. Bunding, J. Phys. Chem. 90 (1986) 1834.

[18] J.M. Hicks, K. Kemnitz, K.B. Eisenthal and T.F. Heınz, J. Phys. Chem. 90 (1986) 560.

[19] Y.R. Shen and P. Ye, Phys. Rev. B28 (1983) 4288.

[20] R.K. Chang, J. Ducuing and N. Bloembergen, Phys, Rev. Letters 15 (1965) 6; J.J. Wynne and N. Bloembergen, Phys. Rev. 188 (1969) 1211

[21] N. Bloembergen and P.S. Pershan, Phys. Rev. 128 (1968) 606.

[22] N. Bloembergen, R.K. Chang, S.S. Jha and C.H. Lee, Phys. Rev. 174 (1968) 813.

[23] Proc. IRE 37 (1949) 1384.

[24] R.C. Miller and W.A. Norland, Phys. Rev. B12 (1970) 4896.

[25] B.F. Levine and C.G. Bethea, J. Chem. Phys. 66 (1974) 3856.

[26] J. Zyss, J. Chem. Phys. 70 (1979) 3333. 